\title{
Genome-wide identification of the expansin gene family reveals that expansin genes are involved in fibre cell growth in cotton
}

\author{
Li-Min Lv ${ }^{1,2}$, Dong-Yun Zuo ${ }^{2}$, Xing-Fen Wang ${ }^{1,3}$, Hai-Liang Cheng ${ }^{2}$, You-Ping Zhang ${ }^{2}$, Qiao-Lian Wang ${ }^{2}$, \\ Guo-Li Song ${ }^{2,4^{*}}$ and Zhi-Ying Ma ${ }^{1,3^{*}}$
}

\begin{abstract}
Background: Expansins (EXPS), a group of proteins that loosen plant cell walls and cellulosic materials, are involved in regulating cell growth and diverse developmental processes in plants. However, the biological functions of this gene family in cotton are still unknown.

Results: In this paper, we identified a total of 93 expansin genes in Gossypium hirsutum. These genes were classified into four subfamilies, including 67 GhEXPAs, 8 GhEXPBs, 6 GhEXLAs, and 12 GhEXLBs, and divided into 15 subgroups. The 93 expansin genes are distributed over 24 chromosomes, excluding Ghir_A02 and Ghir_D06. All GhEXP genes contain multiple exons, and each GhEXP protein has multiple conserved motifs. Transcript profiling and GPCR analysis revealed that the expansin genes have distinct expression patterns among different stages of cotton fibre development. Among them, 3 genes (GhEXPA40, GhEXPA1A, and GhEXPA8h) were highly expressed in the initiation stage, 9 genes (GhEXPA4a, GhEXPA13a, GhEXPA4f, GhEXPA4q, GhEXPA8f, GhEXPA2, GhEXPA8g, GhEXPA8a, and GhEXPA4n) had high expression during the fast elongation stage, and GhEXLA1c and GhEXLA1f were preferentially expressed in the transition stage of fibre development.
\end{abstract}

Conclusions: Our results provide a solid basis for further elucidation of the biological functions of expansin genes in relation to cotton fibre development and valuable genetic resources for future crop improvement.

Keywords: Expansins, Gene family, Fibre, Gene expression profiles, Gossypium hirsutum

\section{Background}

Expansins are a kind of cell wall-loosening protein that are widely present in higher plants, bacteria and fungi. Expansins may unlock the network of wall polysaccharides without lytic activity, permitting turgor-driven cell enlargement [1]. Plant expansins are usually 250 to 275

\footnotetext{
* Correspondence: songguoli@caas.cn; mzhy@hebau.edu.cn

${ }^{2}$ State Key Laboratory of Cotton Biology, Institute of Cotton Research, Chinese Academy of Agricultural Sciences (CAAS), Anyang 455000, China ${ }^{1}$ Hebei Research Base, State Key Laboratory of Cotton Biology in China, Hebei Agricultural University, Baoding 071001, China

Full list of author information is available at the end of the article
}

amino acid residues in length, and the majority have a signal peptide in the $\mathrm{N}$ terminus; the signal peptides are usually 20 to 30 amino acid residues long [2,3]. Typical structures of plant expansins are torpedo-shaped proteins containing two domains, domain I and domain II. Domain I is a six-stranded double-psi beta-barrel (DPBB), which has similar characteristics to the catalytic domain of glycoside hydrolase family 45 (GH45) proteins and contains a conserved His-Phe-Asp (HFD). The DPBB domain does not, however, possess the same catalytic activity as GH45. Domain II is homologous to

(c) The Author(s). 2020 Open Access This article is licensed under a Creative Commons Attribution 4.0 International License, which permits use, sharing, adaptation, distribution and reproduction in any medium or format, as long as you give appropriate credit to the original author(s) and the source, provide a link to the Creative Commons licence, and indicate if changes were made. The images or other third party material in this article are included in the article's Creative Commons licence, unless indicated otherwise in a credit line to the material. If material is not included in the article's Creative Commons licence and your intended use is not permitted by statutory regulation or exceeds the permitted use, you will need to obtain permission directly from the copyright holder. To view a copy of this licence, visit http://creativecommons.org/licenses/by/4.0/ The Creative Commons Public Domain Dedication waiver (http://creativecommons.org/publicdomain/zero/1.0/) applies to the data made available in this article, unless otherwise stated in a credit line to the data. 
group-2 grass pollen allergens [2], and it was recently classified as a family-63 carbohydrate binding module (CBM63) [2, 4].

The plant expansin superfamily is divided into four subfamilies, which include $\alpha$-expansin (EXPA), $\beta$ expansin (EXPB), expansin-like A (EXLA), and expansin-like B (EXLB). Expansins were first identified as endogenous proteins inducing cell wall extension in plants by the McQueen-Mason group [5]. It has been shown that they can participate in many developmental processes and function in cell growth and enlargement, pollen tube invasion of the stigma (in grasses), wall disassembly during fruit ripening, abscission, stress resistance, and other cell separation events $[1,2,6,7]$.

Cotton fibres are single-celled trichomes that differentiate from the ovule epidermis, which is a powerful cell expansion and wall biogenesis research model system [8]. The process of cotton fibre development can be divided into five stages: initiation, elongation, transition, secondary wall synthesis and maturation [9]. Some expansin genes preferentially expressed in cotton fibres were isolated and identified using several different approaches, including cDNA arrays, subtractive PCR, RT-PCR, and so on [10, 11]. The functions of expansin genes in cotton fibre development have been further investigated. Over-expression of GhEXPA8 can improve cotton fibre length and micronaire value [12]. GbEXPATR, a Gossypium barbadense-specific expansin, can also enhance cotton fibre elongation through cell wall restructuring [13]. GhRDL1 is localized in the cell wall and interacts with GhEXPA1, and cotton plants overexpressing GhRDL1 and GhEXPA1 have an increased fibre length and produce many more cotton bolls [14]. GhEXPA1 expression levels are regulated by the transcription factor GhHOX3, which can promote cotton fibre elongation [15].

Thus, expansins play an important role in cotton fibre development. The cotton genome has been sequenced and re-sequenced in succession [16-19]. These genomic data make genome-wide identification of gene families possible. Gossypium hirsutum possesses a complex allotetraploid genome (AADD; $2 \mathrm{n}=52$ ), which resulted from the doubling of two diploid cotton genomes, specifically, those of Gossypium. arboreum (AA; $2 \mathrm{n}=26$ ) and Gossypium raimondii (DD; $2 \mathrm{n}=26)$ [17, 20, 21]. At present, upland cotton accounts for $90 \%$ of natural fibre production worldwide. Hence, we mainly examined the whole expansin gene family of G. hirsutum in this paper. This research can provide genome-wide information on cotton expansin genes and promote further investigations of the biological function of expansin genes during cotton fibre development and other developmental processes.

\section{Results}

\section{Identification and sequence analysis of the cotton expansin gene family}

We have identified the expansin gene family in the $G$. hirsutum genome. As a result, 98 candidate expansin genes were initially obtained. According to the analysis of conserved expansin domains, 93 expansin genes with both DPBB-1 (domain I) and Pollen_allerg_1 (domain II) domains were ultimately identified for further analysis. Each expansin gene was named according to nomenclature guidelines. The detailed results are shown in Table S1. The expansin gene family contained four subfamilies, including EXPA, EXPB, EXLA, and EXLB. For the expansin gene family in G. arboreum and G. raimondii. The same analysis methods were performed. As a result, 49 and 45 expansin genes were identified in the $G$. arboreum and G. raimondii genomes, respectively. These expansin genes were also divided into 4 subfamilies. The detailed results are shown in Table S2 and Table S3.

We analysed the biochemical properties of expansin proteins (Table S1). The pI values of expansin family members ranged from 4.65 (GhEXLB1l) to 12.01 (GhEXPA4c), with an average of 8.47. The pI values of all EXPA and EXLA members were above 7.0, except for those of GhEXPA8b and GhEXPA7d. However, the pI values of all EXPBs and EXLBs were below 7.0, except for those of GhEXPB3a, GhEXPB3b, GhEXPB1a, GhEXPB1b, GhEXLB1d, and GhEXLB1j (Additional file 1: Table S1). The average MW of expansin family members was $27.42 \mathrm{kD}$, ranging from 14.29 (GhEXPA17e) to 41.53 (GhEXPA5e) kD. The length of expansin protein sequences ranged from 150 (GhEXLA17e) amino acids (aa) to 366 aa (GhEXPA5e), and the signal peptide length ranged from 17 (GhEXPA15d, GhEXPA15g and GhEXLA1d) to 35 (GhEXLA1a) aa (Additional file 1: Table S1).

The multiple sequence alignment results of 93 expansin proteins from G. hirsutum showed that they had similar sequence characteristics: the majority of them consisted of a signal peptide and conserved domains I and II (Additional file 2: Figure S1), which was consistent with the findings of a previous study [3]. The amino acid sequence of domain I was more conserved than that of domain II, especially among EXPA members (Additional file 2: Figure S1). Notably, almost all of the EXPAs (excluding GhEXPA13a, GhEXPA13b, and GhEXPA15d) and three EXLA members (GhEXLA17a, GhEXLA17b, and GhEXLA17c) contained a conserved motif (HFD) in domain I (Additional file 2: Figure S1). Members of EXPB, EXLB, and the six other EXLA members did not have the HFD motif. Six EXLAs contained an extra segment named the EXLA extension of the C terminus. The EXLA extension sequence feature was found only in the EXLA subfamily, and the amino acid 
sequences of the EXLA extension were as follows: "DIAK(Q)EGCS(F)P(H)CDD(Y)S(G)H(N)WR(-)". In addition, a conserved motif named BOX 1 was found in almost all the expansin members (Additional file 2: Figure S1).

\section{Phylogenetic relationships, gene structure and protein motifs of cotton expansin genes}

To evaluate the evolutionary relationships of cotton expansins, a phylogenetic tree was constructed. The expansins were divided into four major subfamilies, namely, EXPA, EXPB, EXLA and EXLB. The EXPA subfamily was the largest group, with 67 members, and the other subfamilies contained eight (EXPB), six (EXLA), or 12 (EXLB) members. The four expansin subfamilies comprised 15 subgroups (Fig. 1). We discovered that EXPA-IV was the largest subgroup, which included 17 expansin members, and EXPA-VII, EXPA-VIII, and EXPA-IX were the smallest subgroups, with only two expansin members each.

The results of gene structure (exon-intron organization) analysis showed that the expansin members included two to five exons, and the same subfamilies had similar characteristics of exon types (Fig. 2a, b). Most of the EXPA members had three exons (51 of 67 EXPA members). Twelve EXPAs had two exons, and four EXPAs had four exons. All members of the EXPB subfamily had four exons except for GhEXPB1a (five exons). Four EXLA members contained five exons, and two members had four exons. EXLB members had four (seven $E X L B s$ ) or five exons (five EXLBs).

We identified the conserved motifs in expansin proteins. As a result, a total of ten distinct motifs were identified (Fig. 2c, Additional file 2: Figure S2). The motifs of all cotton expansins had unifying features; for example, each expansin protein contained motif 5 , and all of them contained motif 4, except for GhEXPA15d, GhEXPA17e, and GhEXLB1j. In addition, the type, arrangement, and number of motifs were similar within the same subfamily. More than half of the EXPA members (38/67) had seven motifs, and 21 members had six motifs. The EXPB, EXLA, and EXLB subfamilies possessed similar motif characteristics, and most of them contained five

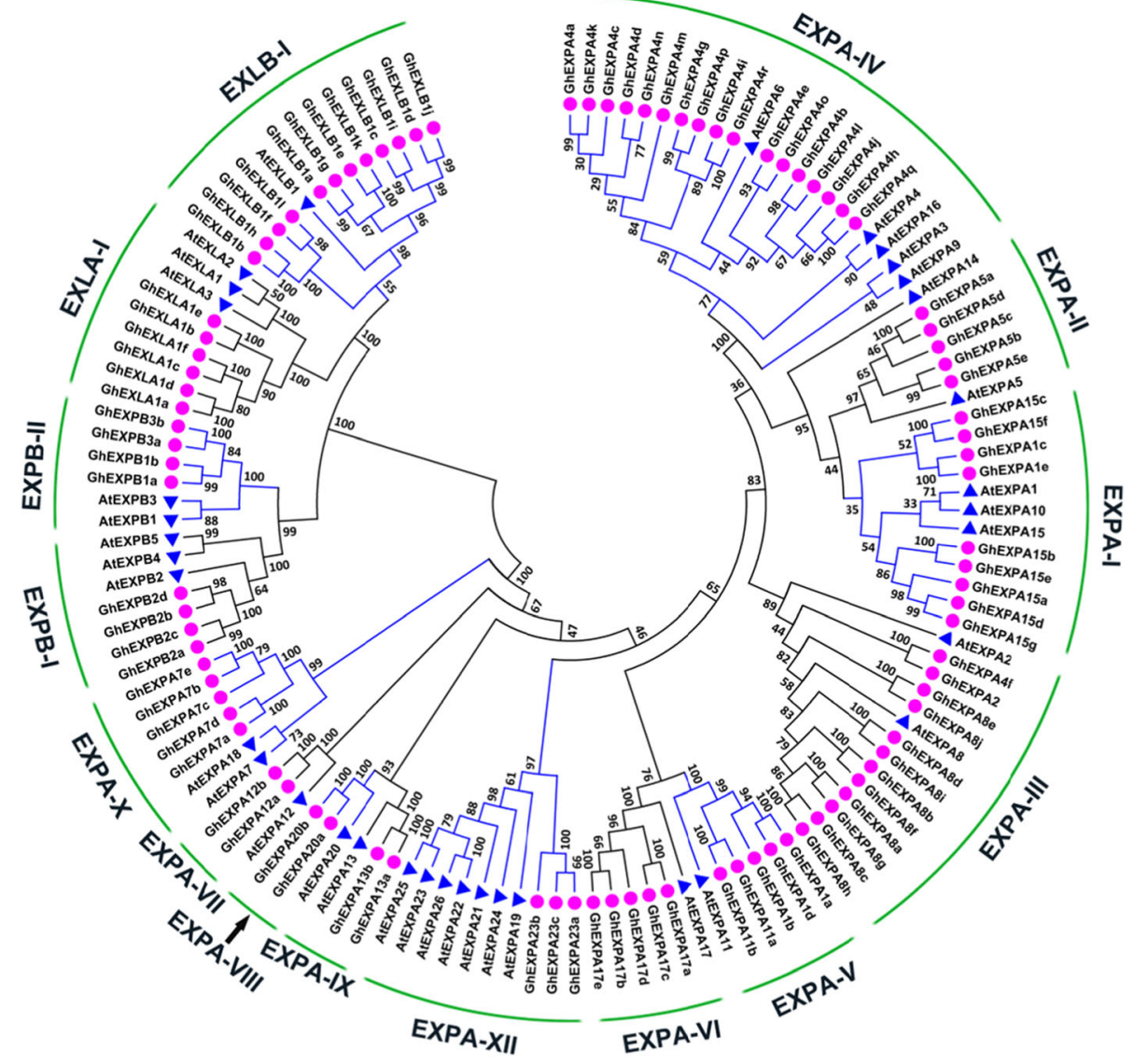

Fig. 1 Phylogenetic analysis and subfamily classification of the expansin genes in cotton (GhEXPs). The phylogenetic tree was constructed with MEGA 6.0 software using the neighbour-joining (NJ) method with 1000 bootstrap replicates. The pink solid circles represent the cotton expansin genes from Gossypium hirsutum; the blue solid triangles represent the expansin genes from Arabidopsis thaliana. Gh, Gossypium hirsutum; At, Arabidopsis thaliana 


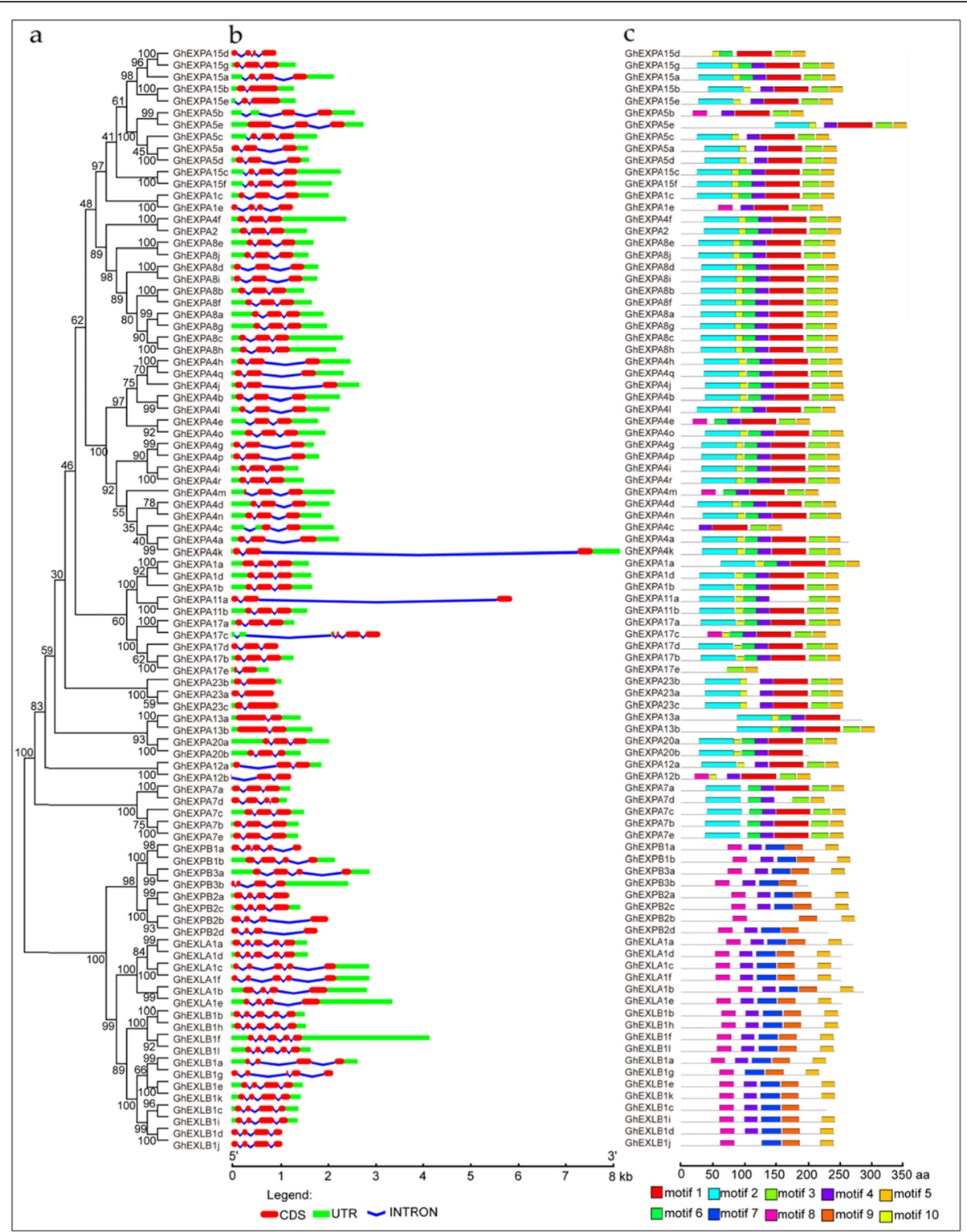

Fig. 2 Phylogenetic relationships, gene structure, and protein domain architecture of GhEXP genes. a Phylogenetic relationships of 93 GhEXP proteins. The phylogenetic tree was constructed with MEGA 6.0 software using the neighbour-joining (NJ) method with 1000 bootstrap replicates. b Gene structure (exon-intron organization) analysis of GhEXPs. The gene structures were drawn online with Gene Structure Display Server 2.0 [22]. The CDSs, introns, and UTRs are marked with red boxes, blue lines, and green boxes, respectively. The scale bar is shown at the bottom. c Analysis of conserved domains of the GhEXP proteins. Differently coloured boxes represent different conserved motifs of GhEXP proteins

motifs (motifs 4, 5, 7, 8, and 9). GhEXPB2d and GhEXPB3b of the EXPB subfamily included four motifs, and three members (GhEXLB1g, GhEXLB1c, and GhEXLB1j) of EXLB also had the same number of motifs. These results showed that the EXPB, EXLA, and EXLB subfamilies had close evolutionary relationships. The similarities between gene structures and sequence motifs implied that cotton expansin family genes underwent duplication over evolutionary time.

\section{Chromosomal location and collinearity analysis of the expansin gene family}

The chromosomal location of GhEXP genes was identified in G. hirsutum. The results are shown in Fig. 3. A total of 93 expansin genes were distributed on 24 chromosomes, excluding Ghir_A02 and Ghir_D06. The chromosome Ghir_A05 contained eight expansin genes, whereas Ghir_A06 included only one expansin gene. The numbers of expansin genes located on other 


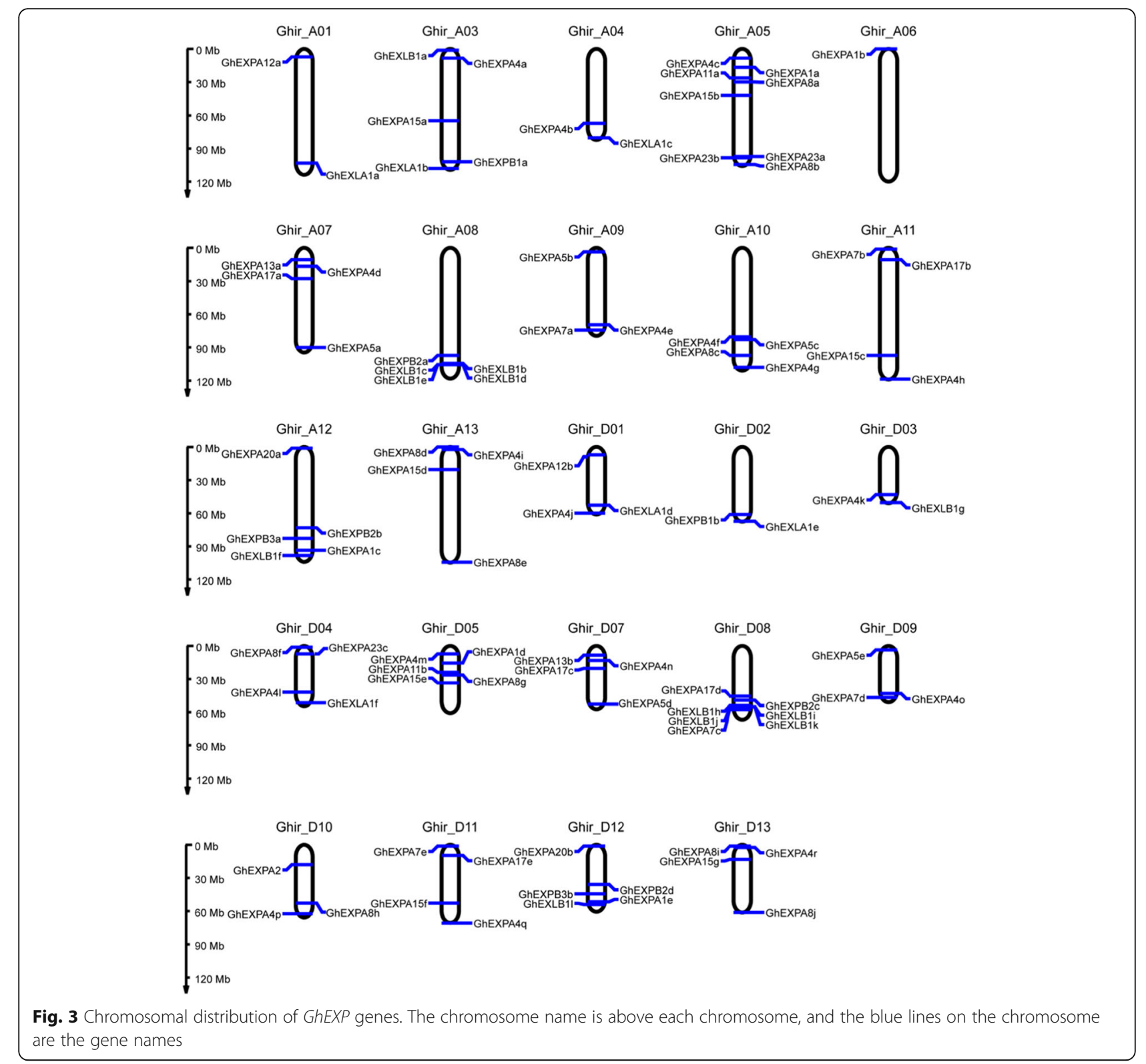

chromosomes ranged from two to seven. In addition, some of the expansin genes were located on the chromosome in clusters; for example, both Ghir_A08 and Ghir_D08 possessed a gene cluster with four distinct EXLBs (Fig. 3). These results showed that the expansin genes were unevenly distributed on each chromosome. Collinearity analysis showed that expansin genes were frequently collinear between the A and D sub-genomes (Fig. 4), which indicated that expansin genes with collinear relationships may have similar functions.

\section{Investigation of cis-acting elements in the promoter regions of expansin genes}

We identified the cis-acting regulatory elements of the cotton expansin gene family. The results showed that the cis-acting regulatory elements of expansin genes were extremely diverse (Additional file 1: Table S4; Table S5). These elements were divided into 7 categories and 111 types, including 31 light-responsive elements, 7 development-related elements, 13 hormone-responsive elements, 5 environmental stress-related elements, 3 promoter-related elements, 7 site-binding-related elements and 44 other elements (no functions). Among them, the light and hormone responsive types were especially abundant (Additional file 1: Table S4; Table S5).

All 93 GhEXP genes possessed 15,200 elements, including 1268 light-responsive elements, 144 developmentrelated elements, 779 hormone-responsive elements, 409 environmental stress-related elements, 9416 promoterrelated elements, 81 site-binding-related elements and 


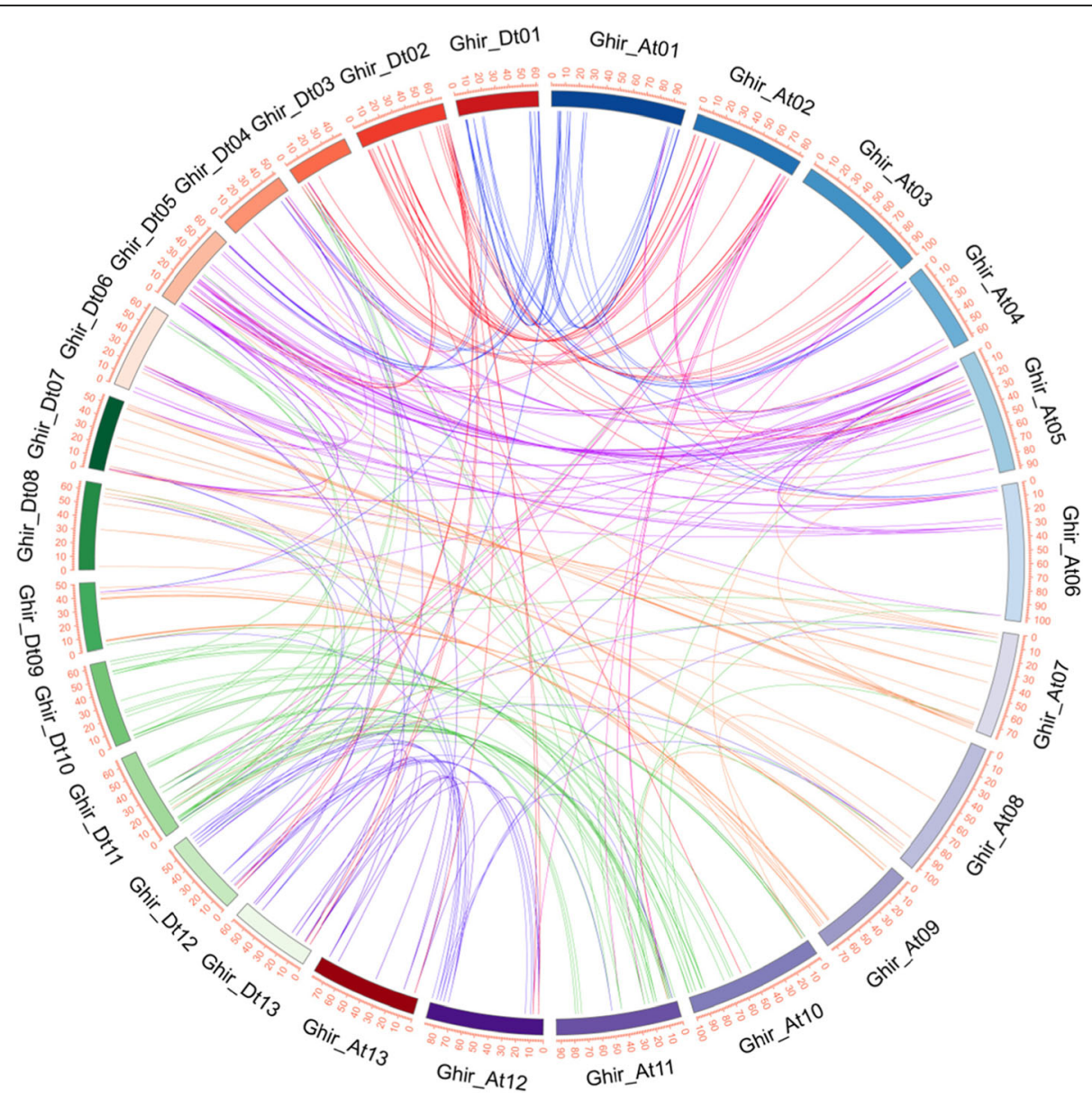

Fig. 4 The collinearity relationships of GhEXP genes in upland cotton. The inner coloured lines show syntenic blocks in homoeologous chromosomes among cotton expansin genes and between the A and D sub-genomes

3103 other elements (Additional file 1: Table S4). Out of 93 GhEXP genes, 83 possessed a Box 4 element (part of a conserved DNA module involved in light responsiveness), 70 possessed a GT1 motif (light-responsive element), 57 had a G-box (cis-acting regulatory element involved in light responsiveness) with 70 enriched ABRE elements (the cis-acting element involved in abscisic acid responsiveness), 75 contained an ERE, 56 had a TGACG motif as well as a TGACG motif, which are the cis-acting regulatory elements involved in MeJA responsiveness, 73 harboured an ARE (cis-acting regulatory element essential for anaerobic induction), and 40 possessed an MBS (MYB binding site involved in drought inducibility). Moreover, these relatively abundant elements were also more conserved among the GhEXP gene family. In addition, all the GhEXP genes contained a CAAT-box and TATAbox, which are the core elements of the promoter in eukaryotes, and these genes contained the largest numbers of these elements (Additional file 1: Table S5).
Expression patterns of the expansin genes in cotton fibre To comprehensively investigate the temporal expression patterns of the cotton expansin gene family, fibre samples at different developmental stages were used for transcriptome analysis. A heat map was constructed with these transcriptome data (Fig. 5). The 86 expansin genes displayed different expression patterns. The remaining seven expansin genes were not detected in the transcriptome data. Although the expression patterns of expansin genes displayed obvious differences, clustered expansin genes generally possessed similar expression patterns. For example, GhEXPA1d, GhEXPA15d, GhEXPA15a, GhEXPA4o, GhEXPA4a and GhEXPA4b were the preferentially expressed genes during the fibre initiation and elongation stages (0 to $15 \mathrm{DPA}$ ), whereas GhEXLA1f and GhEXLA1c had higher expression during the middle and later cotton fibre developmental stages (after 15 DPA). In addition, GhEXPA4f and GhEXPA2, two homologous genes located on the A and D sub-genomes, respectively, were sharply up-regulated from 3 DPA, with very similar expression patterns (Fig. 5), suggesting that they may 


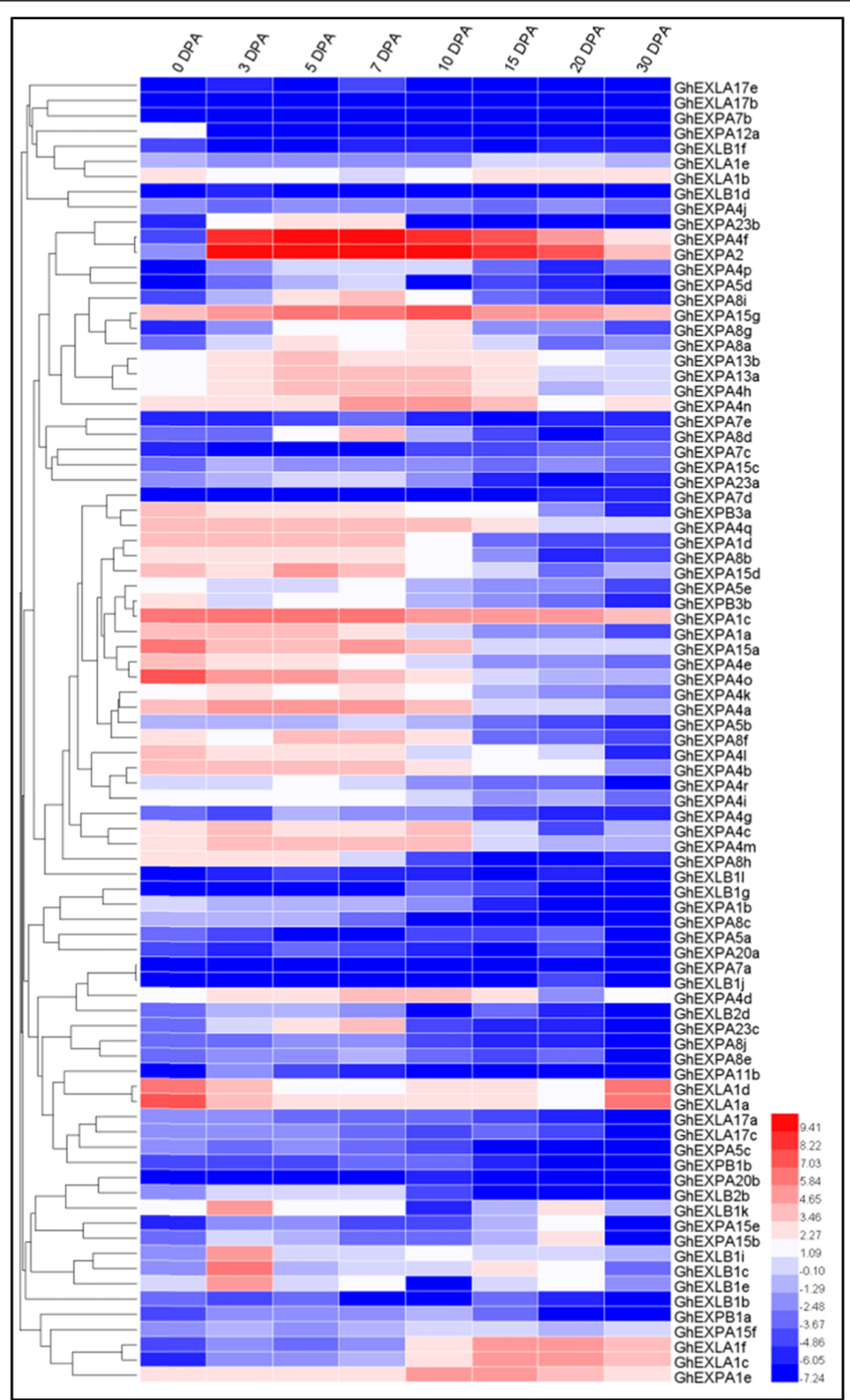

Fig. 5 Expression profiles of GhEXP genes at different cotton fibre developmental stages. The heat map was constructed based on RNA-seg data. Different colours represent the different expression levels of GhEXP genes. The legend represents the logarithm-transformed values of log2. DPA, day post anthesis; FPKM, fragments per kilobase of transcript per million mapped reads 
have similar or complementary functions in cotton fibre development. To verify our transcriptome results, the GhEXP gene expression profiles were further confirmed using publicly available RNA-seq data. The expression profiles of GhEXP genes were generally consistent with our transcriptome results (Fig. 5 and Figure S3).

To avoid missing possible important expansin genes, we also analysed the transcript levels of seven expansin genes that were not detected in the transcriptome data (Fig. 5; Table S1). qRT-PCR showed that the seven expansin genes were scarcely expressed, except for GhEXLB1h, with low expression levels in ovules and fibres. In addition, we found that these genes can be detected in other tissues, but their expression levels were not high (Additional file 2: Figure S4).

\section{qRT-PCR analysis of the special expansin genes in cotton fibres}

To further identify the key expansin genes involved in fibre cell growth, 14 expansin genes that are predominantly expressed in different developmental stages of cotton fibres were selected to verify their expression level using a qRT-PCR experiment. These expansin genes were evidently up-regulated at the initiation, elongation, or transition stage (Fig. 6) and displayed almost consistent expression tendencies when compared to those in the transcriptome data (Additional file 2: Figure S5).

We found that GhEXPA4o, GhEXPA1a, and GhEX$P A 8 h$ were predominantly expressed at 0 DPA (Fig. 6a), suggesting that these three genes may function in the initial developmental stage of fibre cells. Nine expansin genes showed higher expression levels at the fibre elongation stage, with distinct expression characteristics (Fig. $6 \mathrm{~b})$. The expression of GhEXPA4a reached a peak at 3 DPA, and that of GhEXPA13a and GhEXPA4f peaked at 5 DPA. The expression levels of GhEXPA4q, GhEXPA8f, and GhEXPA2 were the highest at 7 DPA, and those of GhEXPA8g, GhEXPA8a, and GhEXPA4n peaked at 10 DPA (Fig. 6b). GhEXPA4f and GhEXPA2 are homologous genes in allotetraploid cotton species that are located in the A and D sub-genomes of the 10th chromosome, respectively, and both genes have specific expression in cotton fibre cells. Moreover, we found that GhEXPA8 $a$ and GhEXPA8g were important genes during cotton fibre elongation. These results revealed that the expression peaks of the majority of genes appeared from 7 to 10 DPA, which are usually called the fast elongation stages. In addition, we obtained two expansin genes that were predominantly expressed at transition stages, named GhEXLA1c and GhEXLA1f (Fig. 6c). Both of them belong to the EXLA subfamily and have unclear biological roles. The expression levels of GhEXLA1c and GhEXLA1f were the highest at $20 \mathrm{DPA}$, which is the transition stage of fibre cells from fast elongation to secondary cell wall synthesis.

To better understand the potential functions of 14 expansin genes, their expression profiles were detected in 11 different tissues, including roots, hypocotyls, stems, leaves, calycles, petals, pollen, stigmas, and fibres at 0 DPA, 10 DPA and 20 DPA. The results showed that these genes presented distinct but partially overlapping expression patterns (Additional file 2: Figure S6).

\section{Discussion}

In this paper, we first reported on the expansin gene family in upland cotton, which included 93 members. All of the genes had two conserved domains, DPBB_1 and Pollen_allerg_1, consistent with results in other plants, such as $A$. thaliana [3], tobacco [22], tomato [23] and Chinese jujube [24]. Therefore, they are typical plant expansin proteins [2]. Phylogenetic analysis revealed that the 93 cotton expansins were divided into 15 subgroups of four subfamilies (Fig. 1). The number of expansin subgroups was consistent with the number of expansin ancestors including 15 to 17 expansin genes, and each of these ancestors evolved into an extant clade in the phylogenetic tree [25]. Thus, we speculated that each clade of the existing cotton expansin family might be extended by each clade ancestor. In addition, cotton expansin genes within every subfamily had structural similarity, and they also showed structural differences among the four subfamilies (Fig. 2b). The structural and evolutionary ancestor characteristics were consistent with those of other plant expansin gene families [22$24]$. In the same subfamily category and even subgroup, most members had almost the same conserved gene structure and motif distribution (Fig. 2 b, c), further confirming their close evolutionary relationships and phylogenetic classification [26].

Our study showed that the EXPA subfamily genes in cotton were significantly expanded, including 67 total EXPAs (Fig. 1); this expansion in EXPAs suggests important functions of this kind of expansin gene in cotton growth and development. Conversely, there were fewer members of the other three expansin subfamilies relative to EXPAs: 8 EXPBs, 6 EXLAs and 12 EXLBs. The proportion of cotton expansin genes in each subfamily was almost consistent with that in other eudicots, such as $A$. thaliana (26 EXPAs, 6 EXPBs, 3 EXLAs, and 1 EXLB), grape (20EXPAs, 4 EXPBs, 1 EXLA, and $4 E X L B s)$, jujube (19 EXPAs, 3 EXPBs, 1 EXLA and 7 EXLBs), and Chinese cabbage (39 EXPAs, 9 EXPBs, 2 EXLAs, and 3 EXLBs) [2, 3, 24, 27, 28]. The proportions are different in monocotyledons, such as rice (33 EXPAs, 18 EXPBs, 4 EXLAs, and 1 EXLB) and maize (36 EXPAs, 48 EXPBs and 4 EXLAs) $[3,29]$, with the most significant difference being that the EXPBs are more numerous in 

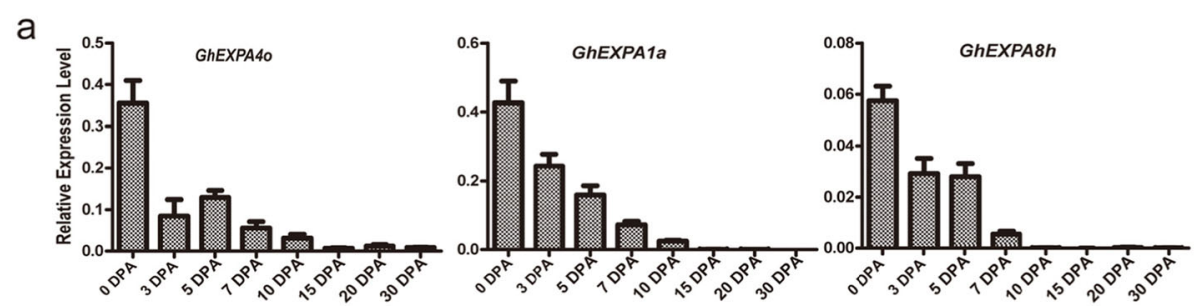

b
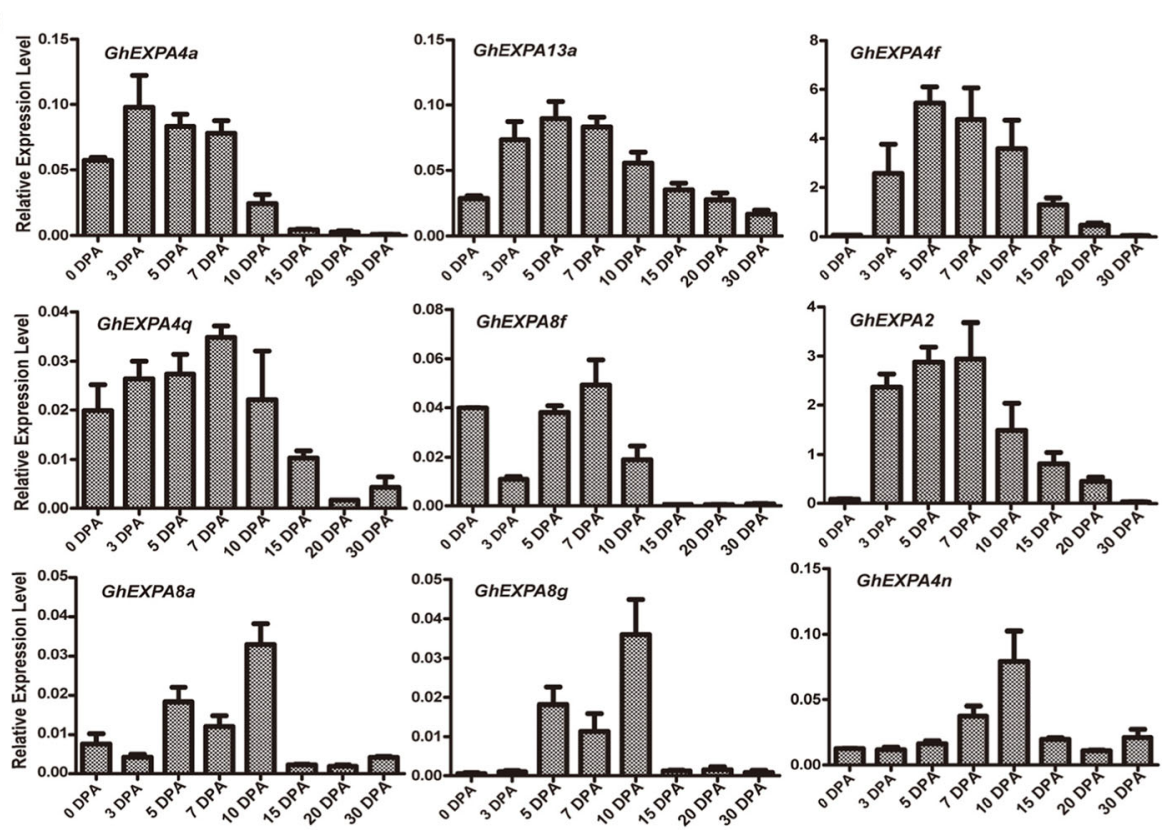

C

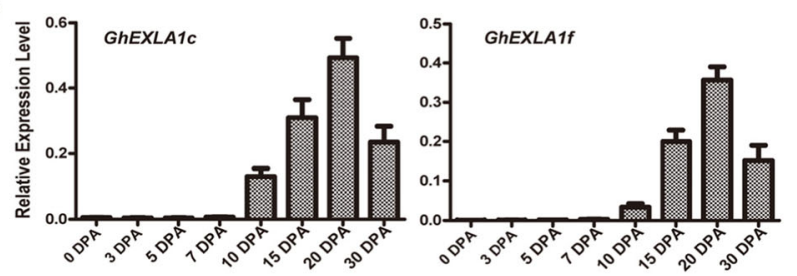

Fig. 6 The expression patterns of 14 GhEXPs at different developmental stages of cotton fibres. a Expression profiles of three GhEXP genes highly expressed in the fibre initiation period. $\mathbf{b}$ Expression profiles of nine GhEXP genes highly expressed in the fibre elongation stage. $\mathbf{c}$ Expression profiles of two GhEXP genes highly expressed at the secondary wall synthesis stage. QRT-PCR experiments were performed with three independent replicates, and the error bars in this figure represent the SDs from three independent experiments

monocotyledons than in eudicots [2]. Furthermore, the number of GhEXP genes (93) was generally consistent with the sum of 49 GaEXPs and 45 GrEXPs. By comparative analysis, we found that 4 expansin subfamilies also existed in G. arboreum (38 EXPAs, 4 EXPBs, 2 EXLAs, and 5 EXLBs) and G. raimondii (33 EXPAs, 4 EXPBs, 3 EXLAs, and 5 EXLBs) (Additional file 1: Table S2; Additional file 1: Table S3). The results indicated that the $\mathrm{A}$ and $\mathrm{D}$ genomes of the two ancestral species were the donors of the modern allotetraploid genome of G. hirsutum [20]. These results provide significant insights into the evolution and functions of expansin genes in cotton.

Based on the expression profiles of expansin genes, we obtained 14 predominantly expressed genes in distinct stages of cotton fibre development, including 12 EXPAs and 2 EXLAs (Fig. 6), excluding EXPBs and EXLBs. Three EXPA genes, GhEXPA4o, GhEXPA1a, and GhEX$P A 8 h$, were first obtained in the early phase of fibre development and displayed high expression levels in qRT-PCR (Fig. 6a); however, their role in the initial stages of fibre development still needs to be clarified. 
Moreover, we also obtained nine expansin genes with higher expression levels in the elongation stages (Fig. $6 \mathrm{~b})$. Among the nine expansin genes, GhEXPA4f and GhEXPA2 had the highest transcriptional levels during cotton fibre development, and they were the most preferentially expressed genes in different tissues (Fig. 6b; Additional file 2: Figure S6b). The expression level of GhEXPA4f was highly consistent with that reported for GhExp1, which was high in the fibre [30]. Its homologous gene GhEXPA2 showed a similar expression pattern, and this result was nearly identical to that for the GhExp2 expression level [30]. Transgenic plants with GhEXPA1 and its partner GhRDL1 exhibit improved cotton fibre yield [14], and overexpression of GhEXPA8 can improve cotton fibre length [12]. By comparative analysis of gene sequences, we confirmed that GhEXPA4f (name in this study, GhirA10G15240), GhExp1 [30] and GhEXPA1 [14] are the same genes, as well as GhEXPA2 (name in this study, GhirD10G12330) and GhEXPA8. Our qRT-PCR results also revealed the importance of the two expansin genes in cotton development. For the other seven new expansin genes, which were predominantly expressed in the elongation stages of cotton fibre development, the functions of these genes in terms of promoting fibre elongation need to be further studied. Interestingly, we found that GhEXPA8a and GhEXP8g are homologous with AtEXP8 in A. thaliana and that AtEXP8 can promote hypocotyl elongation in A. thaliana [31]. This result implies that GhEXPA8a and GhEXP8g can promote fibre cell elongation in cotton. The functional mechanism of GhEXPA8a and GhEXP8g will be one of our important research topics in the future. The above-mentioned expansin genes were the members of the EXPA subfamily that were expressed during the initial and elongation stages of fibre development. This may be because there are more members of the EXPA (67/93) subfamily in the expansin family. Moreover, these data also suggested that expansin genes of the EXPA subfamily are essential in cotton fibre development.

EXLA and EXLB were two smaller expansin subfamilies. Phylogenetic analysis showed that these proteins constitute separate and well-resolved groups; however, their biological functions are uncertain [2]. In this paper, we found two EXLA genes, referred to as GhEXLA1c and GhEXLA1f, with high expression at 20 DPA (Fig. $6 \mathrm{c})$, which is the transition stage of fibre cells from fast elongation to secondary cell wall synthesis. These results suggested that they were important genes during the transition stage, in which cellulose synthesis is performed in preparation for the secondary wall thickening period. At present, there are relatively few studies on EXLA functions besides those of AtEXLA2 in Arabidopsis thaliana. AtEXLA2 was reported to have obvious expression in both the hypocotyl and root; overexpression of AtEXLA2 resulted in slightly thicker walls in non-rapidly elongating etiolated hypocotyl cells [32]. Phylogenetic tree analysis showed that GhEXLA1c, GhEXLA1f and AtEXLA2 were members of the EXLA subfamily (Fig. 1). These results suggested that GhEX$L A 1 c$ and GhEXLA1f could participate in the thickening of the cell wall. In addition, it was reported that an expansin-like protein from Hahella chejuensis could bind cellulose and enhance cellulase activity [33]. It was implied that the two EXLA genes could facilitate cellulose synthesis during the transition; however, the detailed biological functions of EXLAs remain to be assessed in cotton fibre development. More research needs to be conducted in order to understand and make use of EXLAs during cotton transition stages, either in theory or in practice.

\section{Conclusions}

Overall, we successfully performed a genome-scale analysis of the expansin family genes in upland cotton with a special emphasis on fibre development. A total of 93 cotton expansin genes were obtained. Our analysis has provided information for understanding the cotton expansin superfamily, including gene evolution, gene structure, protein motifs, collinear relationships, cis-acting elements and gene expression patterns. Moreover, we obtained the expression patterns of 14 expansin genes in relation to cotton fibre development at different stages. Among them, three genes were highly expressed in the initial stage, nine genes had high-level expression during the fast elongation stage, and GhEXLA1c and GhEXLA1f were preferentially expressed in the transition stage of fibre development. These results lay a foundation for further clarification of the biological functions of expansin genes and the molecular mechanisms of many important agricultural traits in cotton, especially in the elongation stage of cotton fibre development.

\section{Methods \\ Plant materials}

Gossypium hirsutum L. ('TM-1') seeds were obtained from the Institute of Cotton Research of the Chinese Academy of Agricultural Sciences (Anyang, China). TM1 was used as the experimental material in this study. It was planted at the experimental farm $\left(36^{\circ} 06^{\prime} 84.44^{\prime \prime} \mathrm{N}\right.$, $\left.114^{\circ} 49^{\prime} 61.5^{\prime \prime} \mathrm{E}\right)$ of the Institute of Cotton Research of the Chinese Academy of Agricultural Sciences. To research the expression patterns of expansin genes during cotton fibre development, each flower was labelled on the day of flowering, which was considered 0 days post anthesis (DPA). Subsequently, samples were collected at $0,3,5,7,10,15,20$, and 30 DPA. The collected bolls were dissected to obtain ovules and fibres. For 0 to 3 
DPA samples, we collected ovules, and for 5 to 30 DPA samples, we collected fibres. In addition, we also collected cotton tissue samples at different developmental stages, including roots, hypocotyls, stems, and young leaves at the seedling stage and calycles, petals, pollens and stigmas at the adult stage. The different samples were frozen in liquid nitrogen immediately and stored at $-80^{\circ} \mathrm{C}$ in an ultra-low-temperature freezer after harvest.

\section{Identification and sequence analysis of cotton expansin genes}

The cotton genomic data were obtained from the Cotton FGD website [34] (https://cottonfgd.org/). Expansin protein sequences of $A$. thaliana were downloaded from TAIR 10 (http:// www.arabidopsis.org/). First, we used the 35 EXPANSIN protein sequences from $A$. thaliana as queries in searches against the G. hirsutum genome database [19]. BLASTP with default parameters was used to identify the expansin proteins. After that, we searched the database for homologs using "EXPANSIN" as a keyword; finally, we used 6 previously reported GhEXPs as queries to search for other possible GhEXPs by BLASTP searches against the cotton genome [30]. Redundant expansin sequences were deleted after a comparison analysis. Then, all candidate expansin protein sequences were submitted to the NCBI CDD (Conserved Domain Database) (https://www.ncbi. nlm.nih.gov/Structure/cdd/wrpsb.cgi) [35], where conserved domains were identified. To ensure a rapid search speed, we performed this work with the Batch Web CDSearch Tool (https://www.ncbi.nlm.nih.gov/Structure/ bwrpsb/bwrpsb.cgi) [35], where the maximal number of protein queries per request is 4000 , providing adequate processing power for our purposes. We executed the search program using default parameters. The canonical expansin protein contains both conserved domains: DPBB-1 (including the DPBB_1 superfamily) and Pollen_allerg_1 (including the Pollen_allerg_1 superfamily). We acquired the final sequences of the upland cotton expansin family genes for further analysis.

Using the ExPASy online tools [36] (https://www. expasy.org/resources/), we analysed the molecular properties of the identified expansin proteins, which were included to compute the molecular weight (MW) and isoelectric point $(\mathrm{pI})$, and predicted their signal peptide sequences with the SignalP 5.0 server (http://www.cbs. dtu.dk/services/SignalP/). Sequence alignment of the expansin protein sequences was executed in Vector NTI Advance 11 software (version 11.5), followed by searching for the conserved amino acid and domain properties of expansin proteins.

\section{Phylogenetic tree construction}

To analyse phylogenetic relationships, Arabidopsis thaliana (A. thaliana) expansin protein sequences were downloaded from TAIR (https://www.arabidopsis.org/) and EXPANSIN CENTRAL (http://www.personal.psu. edu/fsl/ExpCentral/). Multiple sequence alignment of the identified cotton expansin and A. thaliana expansin proteins was executed in MEGA software (version 6.0) [37], and a phylogenetic tree was constructed with the same software, using the neighbour-joining method. The number of bootstrap replicates was 1000, and the rest of the parameters were set as the defaults.

\section{Analysis of expansin gene structure and motifs}

Analysis of gene structure was performed to identify exons, introns, and UTRs. The corresponding GFF data of identified expansin gene IDs were extracted from the GFF file named Ghirsutum_gene_model in the new cotton genome data [19] (http://cotton.hzau.edu.cn/EN/ download.php), and then the expansin GFF data were analysed using the online tool GSDS (version 2.0, http:// gsds.cbi.pku.edu.cn/) [38]; the results were saved in SVG image format. Motifs of expansin protein sequences were analysed using the online tool MEME (http:// meme-suite.org/index.html) [39]. According to the required file format, we imported the expansin sequences into the online tool. The maximum number of motifs was set to 10 , the repeat number was set to 0 or 1 , and the remainder of the parameters were set to system defaults. The output draft images of gene structure and motif were further modified with Adobe Illustrator CS3 software (version 13.0.0).

\section{Chromosomal locations and collinearity relationships of expansin genes}

We obtained the length of each chromosome from the new genomic data [19], and a file of the lengths of all TM-1 chromosomes was obtained. Then, positional information of the expansin gene on the chromosome was extracted from the GFF file, named Ghirsutum_gene model in the new cotton genome data (http://cotton. hzau.edu.cn/EN/download.php) [19]; thus, a file of positional information of the expansin gene was obtained. Afterwards, the two files were submitted to the online tool MG2C (http://mg2c.iask.in/mg2c_v2.0/) for analysis of expansin gene location on chromosomes. Collinearity analysis of cotton expansin genes was executed by MCScanX software [40], and the visualization of the results was carried out using Circos software [41]. The analysed results were exported in SVG format, and the SVG image was further modified with Adobe Illustrator CS3 software (version 13.0.0).

Analysis of cis-acting regulatory elements in the promoter regions of expansin genes

The promoter regions (2000 bp sequence upstream of the transcription start site in the genomic DNA 
sequence) of the cotton expansin genes were identified by searching the G. hirsutum genome database (https:// cottonfgd.org/) [34], and these promoter sequences were then predicted using PlantCARE (http://bioinformatics. psb.ugent.be/webtools/plantcare/html/) to analyse the cis-acting elements [42].

\section{Transcriptome analysis of cotton expansin genes}

Total RNA was extracted from ovule and fibre samples. Samples from different stages were used for transcriptome analysis with the Illumina platform, including the ovules at 0 and 3 DPA and fibres at 5, 7, 10, 15, 20, and 30 DPA. For transcriptome sequencing, three biological duplicates were conducted for the experimental samples. The sequencing results of gene expression are shown by FPKM (fragments per kilobase of million mapped reads) values. Expression data FPKM values of expansin genes were screened according to the transcriptome results and converted to $\operatorname{logFPKM}$ values. Meanwhile, we downloaded RNA-seq data of cotton ovules and fibres from a public database, the Cotton FGD website (https://cottonfgd.org/) [34], including the ovules at $-3,0,1$, and 3 DPA and fibres at 5, 10, 15, and 20 DPA. The FPKM values were processed as described above. Heatmaps were drawn with the logarithm-transformed values using HemI software (version 1.0) [43].

\section{RNA isolation and real-time quantitative PCR analysis}

The total RNA of samples from different stages was extracted using the RNAprep Pure Kit (for polysaccharide \& polyphenolic-rich plants; cat. no. DP441; Tiangen, Beijing, China). The RNA samples were examined using agarose gel electrophoresis, and then the concentration and quality were analysed with a NanoDrop $\mathrm{ONE}^{\mathrm{c}}$ (Thermo Fisher Scientific, USA). cDNA synthesis was performed on a 2720 thermal cycler (Applied Biosystems, Thermo Fisher Scientific, USA) according to the instructions for the PrimeScript ${ }^{\mathrm{tm}}$ II 1st Strand cDNA Synthesis Kit (TaKaRa, Code No. 6210A). All reversetranscript cDNA samples were diluted 10 times and stored at $-20^{\circ} \mathrm{C}$ for the real-time quantification PCR (qRT-PCR) experiment. The design of specific qRTPCR primers was performed using Beacon Designer software (version 8.0); all the primers are shown in Table S6. qRT-PCRs were performed with the TB Green $^{\text {Tx }}$ Premix Ex Taq ${ }^{\text {Tx }}$ II Kit (TaKaRa, Code No. RR820A) and conducted on a QuantStudio ${ }^{\text {ta }} 5$ RealTime PCR instrument (Applied Biosystems, Thermo Fisher Scientific, USA). UBQ7 (GenBank No. AY189972) was used as a reference gene to calculate relative expression levels. The data were analysed using the $2^{-\Delta \Delta C T}$ method [44].

\section{Supplementary information}

Supplementary information accompanies this paper at https://doi.org/10. 1186/s12870-020-02362-y.

Additional file 1: Table S1. Identification of GhEXP genes in G. hirsutum. Additional file 1: Table S2. The GaEXP genes in G. arboretum. Table S3. The GrEXP genes in G. raimondii. Table S4. Summary of cisacting elements of GhEXP genes. Table S5. The number of cis-acting elements involved in different biological processes in the promoter region of GhEXP genes. Table S6. A list of primers used in qRT-PCR experiments.

Additional file 2: Fig. S1. Multiple sequence alignment of 93 GhEXP proteins. Fig. S2. Analysis of conserved motifs of GhEXP genes in cotton. Fig. S3. Expression profiles of GhEXP genes in cotton ovules and fibres. Fig. S4. Quantitative RT-PCR analysis of seven GhEXP genes in fibres at different stages and in different tissues. Fig. S5. qRT-PCR validation and transcriptome sequencing of 14 GhEXPs at different developmental stages. Fig. S6. Quantitative RT-PCR analysis of 14 cotton GhEXP genes in different tissues.

\section{Abbreviations}

DPA: Days post anthesis; EXPs: Expansins; EXPA: a-expansin; EXPB: $\beta$-expansin; EXLA: Expansin-like A; EXLB: Expansin-like B; DPBB: Double-psi beta-barrel; ERE: Ethylene-responsive element; CDS: Coding sequence

\section{Acknowledgements}

Not applicable.

Authors' contributions

ZM and GS designed the research. $L L, D Z$ and $X W$ performed the research. HC, YZ and QW analysed the data. LL wrote the manuscript. All authors have read and approved the manuscript.

\section{Funding}

This study was financially supported by the National Key Research and Development Program of China (Grant No. 2018YFD0100402) and the National Natural Science Foundation of China (Grant No. 31621005). The funding bodies were not involved in the design of the study; collection, analysis, or interpretation of data; or manuscript writing.

\section{Availability of data and materials}

All of the data and materials supporting our research findings are contained in the methods section of the manuscript. Details are provided in the attached additional files.

Ethics approval and consent to participate Not applicable.

Consent for publication

Not applicable.

\section{Competing interests}

The authors declare that they have no competing interests.

\section{Author details}

${ }^{1}$ Hebei Research Base, State Key Laboratory of Cotton Biology in China, Hebei Agricultural University, Baoding 071001, China. ${ }^{2}$ State Key Laboratory of Cotton Biology, Institute of Cotton Research, Chinese Academy of Agricultural Sciences (CAAS), Anyang 455000, China. ${ }^{3}$ North China Key Laboratory for Crop Germplasm Resources of the Education Ministry, Hebei Agricultural University, Baoding 071001, China. ${ }^{4}$ Zhengzhou Research Base, State Key Laboratory of Cotton Biology, Zhengzhou University, Zhengzhou, China.

Received: 6 September 2019 Accepted: 24 March 2020

Published online: 19 May 2020

\section{References}

1. Cosgrove DJ. Loosening of plant cell walls by expansins. Nature. 2000; 407(6802):321-6. 
2. Cosgrove DJ. Plant expansins: diversity and interactions with plant cell walls. Curr Opin Plant Biol. 2015;25:162-72.

3. Sampedro J, Cosgrove DJ. The expansin superfamily. Genome Biol. 2005; 6(12):242.

4. Georgelis N, Yennawar NH, Cosgrove DJ. Structural basis for entropy-driven cellulose binding by a type-a cellulose-binding module (CBM) and bacterial expansin. Proc Natl Acad Sci U S A. 2012;109(37):14830-5.

5. McQueen-Mason S, Durachko MD, Daniel J. Cosgrove. Two endogenous Pmteins that Induce Cell Wall extension in plants. Plant Cell. 1992;4:1425-33.

6. Wei $P C$, Zhang XQ, Zhao P, Wang XC. Regulation of stomatal opening by the guard cell expansin AtEXPA1. Plant Signal Behav. 2011;6(5):740-2.

7. Li Y, Darley CP, Ongaro V, Fleming A, Schipper O, Baldauf SL, et al. Plant expansins are a complex multigene family with an ancient evolutionary origin. Plant Physiol. 2002;128(3):854-64.

8. Kim HJ, Triplett BA. Cotton Fiber growth in Planta and in vitro. Models for plant cell elongation and Cell Wall biogenesis. Plant Physiol. 2001;127(4): 1361-6.

9. Haigler $\mathrm{CH}$, Betancur L, Stiff MR, Tuttle JR. Cotton fiber: a powerful singlecell model for cell wall and cellulose research. Front Plant Sci. 2012;3:104.

10. An C, Saha S, Jenkins JN, Scheffler BE, Wilkins TA, Stelly DM. Transcriptome profiling, sequence characterization, and SNP-based chromosomal assignment of the EXPANSIN genes in cotton. Mol Gen Genomics. 2007; 278(5):539-53.

11. Ji SJ, Lu YC, Feng JX, Wei G, Li J, Shi YH, et al. Isolation and analyses of genes preferentially expressed during early cotton fiber development by subtractive PCR and CDNA array. Nucleic Acids Res. 2003;31(10):2534-43.

12. Bajwa KS, Shahid AA, Rao AQ, Bashir A, Aftab A, Husnain T. Stable transformation and expression of GhEXPA8 fiber expansin gene to improve fiber length and micronaire value in cotton. Front Plant Sci. 2015;6:838.

13. Li Y, Tu L, Pettolino FA, Ji S, Hao J, Yuan D, et al. GbEXPATR, a speciesspecific expansin, enhances cotton fibre elongation through cell wall restructuring. Plant Biotechnol J. 2016;14(3):951-63.

14. Xu B, Gou JY, Li FG, Shangguan XX, Zhao B, Yang CQ, et al. A cotton BURP domain protein interacts with alpha-expansin and their co-expression promotes plant growth and fruit production. Mol Plant. 2013;6(3):945-58.

15. Shan CM, Shangguan XX, Zhao B, Zhang XF, Chao LM, Yang CQ, et al. Control of cotton fibre elongation by a homeodomain transcription factor GhHOX3. Nat Commun. 2014:5:5519.

16. Zhang T, Hu Y, Jiang W, Fang L, Guan X, Chen J, et al. Sequencing of allotetraploid cotton (Gossypium hirsutum L. acc. TM-1) provides a resource for fiber improvement. Nat Biotechnol. 2015;33(5):531-7.

17. Li F, Fan G, Wang K, Sun F, Yuan Y, Song G, et al. Genome sequence of the cultivated cotton Gossypium arboreum. Nat Genet. 2014;46(6):567-72.

18. Ma Z, He S, Wang $X$, Sun J, Zhang $Y$, Zhang $G$, et al. Resequencing a core collection of upland cotton identifies genomic variation and loci influencing fiber quality and yield. Nat Genet. 2018;50(6):803-13.

19. Wang MJ, Tu LL, Yuan DJ, Zhu D, Shen C, Li JY, et al. Reference genome sequences of two cultivated allotetraploid cottons, Gossypium hirsutum and Gossypium barbadense. Nat Genet. 2019;51:224-9.

20. Li F, Fan G, Lu C, Xiao G, Zou C, Kohel RJ, et al. Genome sequence of cultivated upland cotton (Gossypium hirsutum TM-1) provides insights into genome evolution. Nat Biotechnol. 2015;33(5):524-30.

21. Wang K, Wang Z, Li F, Ye W, Wang J, Song G, et al. The draft genome of a diploid cotton Gossypium raimondii. Nat Genet. 2012;44(10):1098-103.

22. Ding AM, Marowa P, Kong YZ. Genome-wide identification of the expansin gene family in tobacco (Nicotiana tabacum). Mol Gen Genomics. 2016; 291(5):1891-907.

23. Lu YG, Liu LF, Wang X, Han ZH, Ouyang B, Zhang JH, et al. Genome-wide identification and expression analysis of the expansin gene family in tomato. Mol Gen Genomics. 2016;291(2):597-608.

24. Hou L, Zhang Z, Dou S, Zhang Y, Pang X, Li Y. Genome-wide identification, characterization, and expression analysis of the expansin gene family in Chinese jujube (Ziziphus jujuba mill.). Planta. 2019;249(3):815-29.

25. Sampedro J, Lee Y, Carey RE. dePamphilis C, Cosgrove DJ. Use of genomic history to improve phylogeny and understanding of births and deaths in a gene family. Plant J. 2005;44(3):409-19.

26. Zhang S, Xu R, Gao Z, Chen C, Jiang Z, Shu H. A genome-wide analysis of the expansin genes in Malus $x$ Domestica. Mol Gen Genomics. 2014;289(2): 225-36.

27. Krishnamurthy P, Hong JK, Kim JA, Jeong MJ, Lee YH, Lee SI. Genome-wide analysis of the expansin gene superfamily reveals Brassica rapa-specific evolutionary dynamics upon whole genome triplication. Mol Gen Genomics. 2015;290(2):521-30.

28. Dal Santo S, Vannozzi A, Tornielli GB, Fasoli M, Venturini L, Pezzotti M, et al. Genome-wide analysis of the expansin gene superfamily reveals grapevinespecific structural and functional characteristics. PLoS One. 2013;8(4):e62206.

29. Zhang W, Yan H, Chen W, Liu J, Jiang C, Jiang H, et al. Genome-wide identification and characterization of maize expansin genes expressed in endosperm. Mol Gen Genomics. 2014;289(6):1061-74.

30. Harmer SE, Orford SJ, Timmis JN. Characterisation of six alpha-expansin genes in Gossypium hirsutum (upland cotton). Mol Gen Genomics. 2002; 268(1):1-9.

31. Ikeda M, Fujiwara S, Mitsuda N, Ohme-Takagi M. A triantagonistic basic helix-loop-helix system regulates cell elongation in Arabidopsis. Plant Cell. 2012;24(11):4483-97.

32. Boron AK, Van Loock B, Suslov D, Markakis MN, Verbelen JP, Vissenberg K. Over-expression of AtEXLA2 alters etiolated arabidopsis hypocotyl growth. Ann Bot. 2015;115(1):67-80.

33. Lee HJ, Lee S. Ko H-j, Kim KH, Choi I-G. An expansin-like protein from Hahella chejuensis binds cellulose and enhances cellulase activity. Mol Cells. 2010;29(4):379-85.

34. Zhu T, Liang CZ, Meng ZG, Sun GQ, Meng ZH, Guo SD, et al. CottonFGD: an integrated functional genomics database for cotton. BMC Plant Biol. 2017; 17.

35. Marchler-Bauer A, Bo Y, Han LY, He JE, Lanczycki CJ, Lu SN, et al. CDD/ SPARCLE: functional classification of proteins via subfamily domain architectures. Nucleic Acids Res. 2017;45(D1):D200-3.

36. Artimo P, Jonnalagedda M, Arnold K, Baratin D, Csardi G, de Castro E, et al. EXPASy: SIB bioinformatics resource portal. Nucleic Acids Res. 2012;40(Web Server issue):W597-603.

37. Tamura K, Stecher G, Peterson D, Filipski A, Kumar S. MEGA6: molecular evolutionary genetics analysis version 6.0. Mol Biol Evol. 2013;30(12):2725-9.

38. Hu B, Jin J, Guo AY, Zhang H, Luo J, Gao G. GSDS 2.0: an upgraded gene feature visualization server. Bioinformatics. 2015:31(8):1296-7.

39. Bailey $T L$, Boden M, Buske FA, Frith M, Grant CE, Clementi L, et al. MEME SUITE: tools for motif discovery and searching. Nucleic Acids Res. 2009; 37(Web Server issue):W202-8.

40. Wang $Y$, Tang H, Debarry JD, Tan X, Li J, Wang $X$, et al. MCScanX: a toolkit for detection and evolutionary analysis of gene synteny and collinearity. Nucleic Acids Res. 2012;40(7):e49.

41. Krzywinski M, Schein J, Birol I, Connors J, Gascoyne R, Horsman D, et al. Circos: an information aesthetic for comparative genomics. Genome Res. 2009;19(9):1639-45.

42. Lescot M, Dehais P, Thijs G, Marchal K, Moreau Y, Van de Peer $Y$, et al. PlantCARE, a database of plant cis-acting regulatory elements and a portal to tools for in silico analysis of promoter sequences. Nucleic Acids Res. 2002;30(1):325-7.

43. Deng WK, Wang YB, Liu ZX, Cheng H, Xue Y. Heml: A Toolkit for Illustrating Heatmaps. Plos One. 2014;9:11.

44. Livak KJ, Schmittgen TD. Analysis of relative gene expression data using real-time quantitative PCR and the 2(-Delta Delta $C(T))$ method. Methods. 2001;25(4):402-8,

\section{Publisher's Note}

Springer Nature remains neutral with regard to jurisdictional claims in published maps and institutional affiliations.

Ready to submit your research? Choose BMC and benefit from:

- fast, convenient online submission

- thorough peer review by experienced researchers in your field

- rapid publication on acceptance

- support for research data, including large and complex data types

- gold Open Access which fosters wider collaboration and increased citations

- maximum visibility for your research: over $100 \mathrm{M}$ website views per year

At BMC, research is always in progress.

Learn more biomedcentral.com/submissions 